\title{
Complete transition from open to laparoscopic living donor hepatectomy: 8-year experience with more than 500 laparoscopy
} cases

\author{
Jinsoo Rhu, Gyu-Seong Choi, Jong Man Kim, Jae-Won Joh
}

Department of Surgery-Transplantation, Samsung Medical Center, Seoul, Korea

Background: We designed this study to comprehensively review the laparoscopic living donor liver transplantation of our institution.

Methods: Living donor liver transplantation cases performed since the first laparoscopic living donor hepatectomy, until reaching 500th laparoscopic cases were reviewed. Laparoscopic cases were compared to open cases in a yearly basis, regarding the donor selection, donor morbidity, recipient morbidity and operation time.

Results: During the period of May 2013 to July 2021, 754 living donor liver transplantations, 506 laparoscopic, and 247 open cases were performed. Complete transition to laparoscopy was achieved in 2020, performing 112 transplantations laparoscopically. Variation of bile duct type of donor became similar in $2018(\mathrm{P}=1.000)$. There were no differences in the occurrence of grade III complication of donor and recipient throughout the study period. Mean donor operation time were significantly longer in the laparoscopy group which became similar since $2017(\mathrm{P}=0.313)$. There were no differences in the mean operation time of recipients throughout the study period. Regarding graft survival and overall survival of the recipient, there were no difference between the two group throughout the period.

Conclusions: In the initial period, donor selection existed especially for bile duct variation maintaining the safety of the donor and recipient. However, with accumulated experience, complete transition to laparoscopy became possible after 7 years of practice.

This is an Open Access article distributed under the terms of the Creative Commons Attribution Non-Commercial License (http://creativecommons.org/licenses/by-nc/4.0/) which permits unrestricted non-commercial use, distribution, and reproduction in any medium, provided the original work is properly cited. 